\title{
Publisher's Note: Tunneling Dynamics in Multiphoton Ionization and Attoclock Calibration [Phys. Rev. Lett. 114, 083001 (2015)]
}

Michael Klaiber, Karen Z. Hatsagortsyan, and Christoph H. Keitel (Received 3 August 2015; published 12 August 2015)

This paper was published online on 23 February 2015 with a duplicate of Eq. (5) in place of Eq. (6). Equation (6) should read as $\Gamma \sim \exp \left[2 i\left(1+\gamma^{2} / 5\right) \int_{x_{i}}^{x_{e}} p_{x}(t(x)) d x\right]$. The equation has been corrected as of 29 July 2015 . The equation is incorrect in the printed version of the journal. 